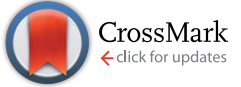

Cite this: RSC Adv., 2017, 7, 6724

Received 21st November 2016 Accepted 11th January 2017

DOI: 10.1039/c6ra27102b

www.rsc.org/advances

\section{Hybrid luminescent porous silicon for efficient drug loading and release $\uparrow$}

\author{
A. Ghafarinazari, ${ }^{a}$ M. Scarpa, ${ }^{b}$ G. Zoccatelli, ${ }^{c}$ M. Comes Franchini, ${ }^{d}$ E. Locatelli*d \\ and N. Daldosso*a
}

In recent decades, biocompatible and light emitting porous silicon (pSi) showed the possibility for use in biomedical applications. Unfortunately, until now the biological applications of light-emitting pSi have been severely limited due the immediate surface oxidation of this nanostructured material, followed by loss of optical properties and degradation in aqueous solutions. In view of drug delivery, the red-ox activity of $\mathrm{pSi}$ damages the carried drug. Herein, we have investigated the stabilization of $\mathrm{pSi}$ microparticles in aqueous solution by covalent attachment of polymers to a short-chain organic coating grafted onto inorganic silicon. The morphological, chemical and optical stability obtained using polyethylene glycol confirm this biocompatible polymer as a superior shell matrix nanostructure for use in biological environments

\section{Introduction}

Space and time controlled delivery of drugs is a pharmacological challenge since it would reduce the waste and the side effects of drugs, thus improving their therapeutic properties. ${ }^{\mathbf{1 , 2}}$ Drug delivery systems (DDS) should offer enough room for drug loading, provide an internal surface with an affinity for the cargo and penetrate homogeneously and efficiently the target tissue or barrier. Indeed, DDS control the release kinetics of the drug in the desired location. In this regard, porous materials are interesting because they offer the potential to reduce systemic toxicity and increase the amount of loaded drug. ${ }^{3}$ Among them, porous silicon (pSi) appears a suitable material for the realization of DDS because of: (i) its large pore volume, ${ }^{4}$ (ii) its "tuneable" surface chemistry, ${ }^{5}$ and (iii) its low toxicity associated with safe biodegradation pathways. ${ }^{6}$ Porous silicon has

${ }^{a}$ Department of Computer Science, University of Verona, Strada le Grazie 15, 37134 Verona, Italy.E-mail: nicola.daldosso@univr.it

${ }^{b}$ Department of Physics, Istituto Nazionale Biostrutture Biosistemi, University of Trento, Via Sommarive 14, 38123 Trento, Italy

${ }^{c}$ Department of Biotechnology, University of Verona, Strada le Grazie 15, 37134 Verona, Italy

${ }^{d}$ Department of Industrial Chemistry, University of Bologna, Viale Risorgimento 4, 40136 Bologna, Italy. E-mail: erica.locatelli2@unibo.it

$\dagger$ Electronic supplementary information (ESI) available: Fig. S1. Schematic representation of the pSi surface functionalization procedure. Fig. S2. HPLC trace of compounds released from hydroxocobalamin (OH-Cbi) by UV detection at 168-351 nm (a). Optical density at times of $289 \mathrm{~s} \mathrm{(b)} \mathrm{and} 380 \mathrm{~s} \mathrm{(c).} \mathrm{Fig.} \mathrm{S3.}$ Optical emission spectra of pSi-chitosan sample after suspension in PBS as a function of time. Fig. S4. (a) Influence of Cbi loading on reduction of pSi-COOH PL intensity into PBS. (b) PL spectrum of $\mathrm{pSi}-\mathrm{COOH}$ initially and after $3 \mathrm{~h}$ of incubation in PBS, and effect of Cbi loading. See DOI: 10.1039/c6ra27102b been investigated by many groups as DDS and still is a field of intense research. ${ }^{7-11}$ Moreover, well assessed fabrication protocols produce pSi microparticles with intense photoluminescence (PL) in the visible region, which can be tracked in cells by fluorescence imaging in real time. ${ }^{12,13}$ Another promising application of pSi for medicine is photo-thermal therapy, which is based on absorption of light and on its transformation to heat in order to kill the target (cell, tissue). ${ }^{\mathbf{1 4}}$ These properties make pSi particles based systems particularly suitable for both therapy and diagnosis of diseases, allowing for real-time monitoring of the therapeutic efficacy and for a reduction of drugs administration, which are the basis of the novel and promising field of "theranostic". ${ }^{15}$

However, un-resolved questions still limit the effective utilization of the pSi as delivery system: (a) the fast degradation of pSi in in vitro conditions ${ }^{\mathbf{1 6}}$ and in harsh biological environments, ${ }^{17}$ (b) the irreversible quenching of the pSi luminescence due to interaction with water or other biomolecules, ${ }^{18}$ (c) the decomposition of a carried drug by redox activity of $\mathrm{pSi}^{\mathbf{1 9}}$

One of the main applications of pSi is for bioimaging due to light emission in the biological window (BW). Absorption of $\mathrm{pSi}$ in BW is quite low, but this issue can be solved, for example, by using two photon absorption (TPA). Indeed, instead of using typical excitation at 350 or $400 \mathrm{~nm}$, by means of TPA (with excitation wavelength of 700 or $800 \mathrm{~nm}$, which perfectly matches BW) it is possible to get efficient light emission thus being used in bioimaging. ${ }^{20}$ The other issue is the optical stability of pSi in biological media, e.g. water based solutions. In fact, while pSi covered by a shell of acrylic groups ( $\mathrm{pSi}-\mathrm{COOH}$ functionalization) introduced by a hydrosilylation reaction shows efficient PL in ethanol for very long time, ${ }^{21}$ a stable light emission in biological media, such as phosphate-buffered 
saline (PBS) solution, still remains a big challenge. ${ }^{\mathbf{1 6}}$ Some protocols have been proposed to postpone pSi degradation up to hours, for example: thermal oxidation resulted in $4 \mathrm{~h}$ stability ${ }^{12}$ while silica coating led to $8 \mathrm{~h}$ stability. ${ }^{22}$ Another example for PL stability is the reaction of pSi with borax, which increased the half-life from less than one day to 2 days. $^{23}$ Previous published protocol with PEG obtained 100\% dissolution in PBS just in 3 days. ${ }^{17}$

Moreover, recently rapid thermal oxidation succeeded in stabilization for about 5 days. ${ }^{6,24}$ Then, optical stability of pSi in biological media remains an issue for application in biomedicine. Oxidation mechanisms of freshly pSi in aqueous solutions have been already deeply investigated by Ogata et $a .^{25}$ The problem of nanostructure stability in biological environment is however quite general. Nanomaterials, in fact, can often present chemical un-stability due to the large exposed surface, but also biological reactivity, since they undergo opsonisation and uptake by cells of the immune system. ${ }^{12}$ Opportune modification of the surface enables limiting these effects, meanwhile conferring desired properties to the final product. ${ }^{26}$

Among all the possibilities, the use of polyethylene glycol (PEG) has been shown appealing for applications in biomedical field. Indeed, PEG can ensure water stabilization to the nanosystems, ${ }^{27}$ meanwhile being biocompatible and able to reduce, once in vivo, opsonisation phenomena, which usually are the main factor affecting particles circulation and earlier elimination from the bloodstream. ${ }^{28}$ PEG is not the only polymer used for this purpose; recently, biopolymers derived from natural sources have showed promising and interesting capacity. ${ }^{29}$ Among others, chitosan is the product of the de-acetylation of chitin, the second most abundant biopolymer in the world generally found in crustacean shells. $^{30}$ It is also used to form nano or micro water-soluble particles able to incorporate drugs or active agents. ${ }^{31}$ Finally, both PEG and chitosan, due to their carboxylic and amino groups respectively, offer the possibility of being linked to biomolecules (peptides, proteins, monoclonal antibodies and aptamers) thus providing to the final DDS potential targetable features.

On the other hand, the interaction between pSi and drugs must be carefully considered to design an effective delivery system without any side effect on the drug itself. As already reported, $\mathrm{pSi}$ in biological systems induces a strong redox activity. ${ }^{5}$ In fact, oxidation of pSi produces free electrons that can reduce drugs, ${ }^{32}$ proteins, ${ }^{33}$ and cells components. ${ }^{34}$ It is well known that the oxidation of pSi can reduce water to hydrogen according to reaction (1) with a large Gibbs energy, and in general, standard electrode potential of silicon is about $1.697 \mathrm{~V}^{35}$ Thus, modification of pSi surface is needed to both limit the degradation of drugs due to their reduction and to increase the affinity of the drug for the pSi surface.

$$
\mathrm{Si}+2 \mathrm{H}_{2} \mathrm{O} \rightarrow \mathrm{SiO}_{2}+2 \mathrm{H}_{2}\left(\Delta G^{\circ}=-382 \mathrm{~kJ}\right)
$$

In the literature, the most used way to modify pSi surface, in order to avoid drug reduction, is oxidation. Sailor et al. ${ }^{19,32,36}$ proposed complete oxidation at high temperature $\left(800{ }^{\circ} \mathrm{C}\right)$ to reach this goal; in fact, the silicon skeleton was completely oxidized to ensure the drug not to be reduced or degraded by contact with elemental silicon during the particle dissolutiondrug release phase. On the contrary, Barnes et al. ${ }^{33}$ proposed a mild oxidation at $400{ }^{\circ} \mathrm{C}$ to avoid reduction of proteins. Based on free kinetics model, ${ }^{37}$ we proposed that mild oxidation leads to remove surface moieties (such as hydroxyl agents) and the surface oxidation is obeying Avrami-Erofeev model. By increasing the oxidation conditions, i.e. longer time and temperature, oxygen diffuses through oxidized surface layer to oxidize the underlying silicon according to 3D diffusion model.

However, oxidation quenches the visible light emission of $\mathrm{pSi}^{38}{ }^{38}$ which is fundamental for bioimaging ${ }^{39}$ and photo-thermal therapy. ${ }^{\mathbf{1 4}}$ Moreover, the oxidation induces a reduction of the pores volume because of the swelling of the pore walls thus significantly reducing the drug loading amount. As an example, oxidation at $400{ }^{\circ} \mathrm{C}$ and $800{ }^{\circ} \mathrm{C}$ led to 3-fold and 10-fold reduction of loading capacity of human serum albumin ${ }^{33}$ and cobinamide, ${ }^{36}$ respectively.

In the present study, we have addressed the possibility of using surface coating to control redox activity of pSi microparticles in biological systems thus maintaining the light emission properties for long time. Moreover, by using cobinamide, which is a vitamin 12 analogue and behaves as a probe of the redox activity of $\mathrm{pSi}^{36}$ we investigated the effect of the surface chemistry of pSi on drug stability, loading amount and release profile.

\section{Experimental procedure}

All chemicals were purchased from Sigma-Aldrich and used as received (all ACS grade). All aqueous solutions were prepared with ultrapure water obtained by using an ultrafiltration system (Milli-Q, Millipore) with resistivity above $20 \mathrm{M} \Omega \mathrm{cm}^{-1}$.

\subsection{Preparation of $\mathrm{pSi}-\mathrm{COOH}, \mathrm{pSi}-\mathrm{NH}_{2}$ and $\mathrm{pSiO}_{2}$ microparticles}

Porous silicon layer was formed by electrochemical etching of p-type Si wafer, (100) oriented, with 10-20 $\Omega \mathrm{cm}$ resistivity (University wafers, Boston MA). The etching was done at $80 \mathrm{~mA}$ $\mathrm{cm}^{-2}$ constant current for $5 \mathrm{~min}$ in $2: 1$ ethanol : $\mathrm{HF}(48 \%)$ solution. The porous silicon layer was sonicated for $15 \mathrm{~min}$ in anhydrous toluene $(80 \mathrm{~mL})$ under nitrogen atmosphere to get pSi microparticles. To introduce the carboxylic $(\mathrm{COOH})$ or amine functionalities $\left(\mathrm{NH}_{2}\right)$ on the pSi surface, the so prepared pSi was sonicated in toluene containing NHS ester of $5 \mathrm{mM}$ acrylic acid (pSi-CONHS). Then, to allow the hydrosilylation reaction to occur, the so obtained pSi powder suspension was illuminated with white light $(250 \mathrm{~W})$ for $2 \mathrm{~h}$, under argon atmosphere. After this step, the pSi-CONHS powder was centrifuged and rinsed with toluene. To remove the NHS group and obtain microparticles exposing the free carboxylic group (pSi-COOH), the NHS group was hydrolysed in ethanol and then the powder was rinsed several times with the same solvent.

To obtain microparticles exposing amino groups ( $\mathrm{pSi}-\mathrm{NH}_{2}$ ), the pSi-COOH powders were suspended in toluene and left to react with trioxa-1,13-tridecanediamine, under gentle shacking. Dicyclohexylcarbodiimide $(200 \mu \mathrm{M})$ was added to increase the reaction yield. Morphological and optical properties of these 
samples were already studied in detail. ${ }^{21}$ When necessary, the pSi powders were dried removing the solvents by a gentle nitrogen flow in order to avoid air oxidation. The aging of pSi in air in our pSi samples and the related PL modifications have been already studied in the past. ${ }^{40}$

In this paper, the main goal is to compare pSi microparticles with the different surface coatings, as previously described, with completely oxidized pSi microparticles presented in literature. These oxidized porous silicon $\left(\mathrm{pSiO}_{2}\right)$ microparticles were obtained by oxidation of $\mathrm{pSi}$ at $1000^{\circ} \mathrm{C}$ for $10 \mathrm{~min}$ by heating rate of $3 \operatorname{min~} \mathrm{K}^{-1} \cdot{ }^{37}$

\subsection{Coating of $\mathrm{pSi}-\mathrm{COOH}$ microparticles with PEG or chitosan}

As for pSi coated by PEG (pSi-PEG1), polyethylene glycol with amino and carboxylic acid end groups $\left(\mathrm{HCl} \cdot \mathrm{H}_{2} \mathrm{~N}-\mathrm{PEG}-\mathrm{COOH}\right.$, MW $\sim 3 \mathrm{kDa}$ ) was purchased from Rapp Polymere $\mathrm{GmbH}$ (Tübingen, Germany). First, pSi-COOH (0.3 mg) were dispersed in anhydrous tetrahydrofuran (THF, $1 \mathrm{~mL}$ ). Carbonyldiimidazole (CDI, $0.8 \mu \mathrm{mol}$ ) was added to the reaction mixture under nitrogen. After $90 \mathrm{~min}, \mathrm{HCl} \cdot \mathrm{H}_{2} \mathrm{~N}-\mathrm{PEG}-\mathrm{COOH}(50 \mathrm{mg}, 1.6 \mu \mathrm{mol})$ dissolved in $1 \mathrm{~mL}$ of THF and diisopropylethylamine (DIPEA, $10 \mu \mathrm{L}, 50 \mu \mathrm{mol}$ ) were added. The reaction was stirred in vortex for 24 hours at room temperature. After that, particles were collected in centrifuge (13 $100 \mathrm{rpm}, 4 \mathrm{~min}$ ), washed twice with $2 \mathrm{~mL}$ of a mixture $1: 1$ THF : water and centrifuged again before dispersion in ultrapure water $(1 \mathrm{~mL})$. Schematic passages of this reaction were shown in path A in Fig. S1a on the ESI. $\dagger$ In order to better understand the effect of PEG on pSi, the ratio of PEG/pSi was increased 2 and 3 times (samples pSi-PEG2 and pSi-PEG3, respectively). Moreover, native PEG (MW $\sim 3 \mathrm{kDa}$ ) was used as a reference.

In the case of chitosan functionalization (pSi-chitosan), to a solution of the $\mathrm{pSi}-\mathrm{COOH}$ in ethanol $(1 \mathrm{~mL}, 0.3 \mathrm{mg})$, chitosan dissolved in acetate buffer $\left(\mathrm{pH}=4.3,0.25 \mathrm{mg} \mathrm{mL}{ }^{-1}, 1.25 \mathrm{mg}\right.$ ) was added drop wise. The system was left to incubate with mechanical stirring for 24 hours. After that, particles were collected in centrifuge (13 $100 \mathrm{rpm}, 4 \mathrm{~min}$ ), washed twice with 2 $\mathrm{mL}$ of water and centrifuged again before re-dispersion in ultrapure water $(1 \mathrm{~mL})$. Schematic passages of this reaction were shown in path B in Fig. S1b. $\uparrow$ Table 1 summarizes the different samples investigated in this work.

\subsection{Synthesis of cobinamide}

Cobinamide was synthesized from hydroxocobalamin $(\mathrm{OH}-\mathrm{Cbi}$, purchased from Sigma-Aldrich) by acid hydrolysis using $\mathrm{HCl}$ as

Table 1 Components used in this research

$\mathrm{pSi} \quad$ Native porous silicon

$\mathrm{pSiO}_{2} \quad$ Thermally oxidized $\mathrm{pSi}$

pSi-COOH pSi functionalized to expose carboxyl group

pSi-PEG1 pSi-COOH functionalized with PEG

pSi-PEG2 pSi-COOH functionalized with double amount of PEG

pSi-PEG3 pSi-COOH functionalized with triple amount of PEG

PEG Native PEG polymer

$\mathrm{pSi}-\mathrm{NH}_{2} \quad \mathrm{pSi}-\mathrm{COOH}$ functionalized by diamine

pSi-chitosan pSi-COOH functionalized with chitosan proposed by Broderick et al. ${ }^{41}$ Purity of Cbi was confirmed both spectrophotometrically by comparison with published spectra $^{\mathbf{4 2 , 4 3}}$ and by high performance liquid chromatography (HPLC) by using a C18 reversed phase column eluted isocratically in $100 \mathrm{mM} \mathrm{NaH}_{2} \mathrm{PO}, \mathrm{pH} 4.0$, and $15 \%$ methanol (v/v). ${ }^{44}$ The column effluent was monitored at multiple wavelengths (168$351 \mathrm{~nm}$ ) by a diode array detector. Fig. S2 $\uparrow$ shows HPLC results, which confirmed at $380 \mathrm{~s}$ that Cbi was obtained and had similar optical density with the references. When stored at $-20{ }^{\circ} \mathrm{C}, \mathrm{Cbi}$ was spectroscopically stable for at least one month.

\subsection{Structural investigation}

$\zeta$-Potential of the samples was measured by the Zetasizer Nano$\mathrm{S}$ (Malvern) instrument, working with a $532 \mathrm{~nm}$ laser beam, and clear disposable $\mathrm{Z}$ cells. The results were expressed as an average of three measurements. The Phase Analysis Light Scattering principle was used to quantify the $\zeta$-potential and electrophoretic mobility. The concentration of the samples was maintained at $\sim 0.1 \mathrm{wt} \%$ and the experiment was conducted at $25{ }^{\circ} \mathrm{C}$. Diluted solutions of $\mathrm{NaOH}$ and $\mathrm{HCl}$ were utilized for making $\mathrm{pH}$ variation.

The surface chemistry was investigated by Fourier Transform Infra-Red (FTIR) spectroscopy. The spectra were acquired by a micro-FTIR Nicolet iN10 instrument, in the spectral range of $700-4000 \mathrm{~cm}^{-1}$ with about $4 \mathrm{~cm}^{-1}$ resolution. Furthermore, scanning electron microscopy images were acquired with a SEM Zeiss instrument EVO 50' EP.

\subsection{Emission stability in biological media}

In order to check workability of the samples as bioimaging agent, photoluminescence (PL) measurements were performed by Horiba Jobin-Yvon Nanolog instrument. The configuration setup was: excitation at $350 \mathrm{~nm}, 2 \mathrm{~nm}$ slit size, $1200 \mathrm{~g} \mathrm{~mm}^{-1}$ density grating (blazed at $500 \mathrm{~nm}$ ), cut-off filtration at $370 \mathrm{~nm}$, and 0.1 second integration time.

To estimate the light efficiency of the samples, quantum yield $(\Phi)$ measurements have been carried out by the comparative method. ${ }^{45,46}$ In this regard, rhodamine 101 was selected as reference, having about $100 \%$ quantum yield. ${ }^{19}$ The quantum yield of a sample was calculated via $\Phi_{\mathrm{s}}=\Phi_{\mathrm{r}} \cdot I_{\mathrm{S}} / I_{\mathrm{r}} \cdot A_{\mathrm{r}} / A_{\mathrm{s}} \cdot\left(n_{\mathrm{s}} / n_{\mathrm{r}}\right)^{2}$, where $I$ is the integrated emission intensity, $A$ is the absorbance, and $n$ is the refractive index of the buffer solution. The subscript $\mathrm{s}$ and $\mathrm{r}$ refer to the sample and the reference solutions, respectively. The samples were further diluted to keep the absorbance less than 0.1 in order to minimize non-uniform irradiation. $^{47}$

Phosphate-buffered saline (PBS, $\mathrm{pH}$ 7.4) was used as simulator of biological fluids. About $30 \mu \mathrm{g}$ from each dried sample was suspended in $3 \mathrm{~mL}$ of PBS, at a concentration of about $10 \mu \mathrm{g}$ $\mathrm{mL}^{-1}$. Then, PL spectrum of the samples was monitored as a function of time.

\subsection{Interaction of pSi with Cbi}

To load Cbi into pSi, we used the procedures already proposed in the literature. ${ }^{36,48} \mathrm{~A}$ small amount of dried pSi (0.5-1 mg) admixed with the same amount of Cbi, was incubated for $2 \mathrm{~h}$ in 
$1.5 \mathrm{~mL}$ of deionized water at $\mathrm{pH} 6$ (adjusted by $\mathrm{HCl}$ ), under weak shaking. Cbi loaded particles were then washed three times with water, recollected by centrifugation and dried in freezedryer. Then, the powder was dispersed in $1 \mathrm{~mL}$ of aqueous PBS.

As for the loading capacity and release rate studies, pSi microparticles loaded with Cbi were dispersed in $1 \mathrm{~mL}$ of PBS at a particle concentration of $100 \mu \mathrm{g} \mathrm{mL}^{-1}$ at room temperature for $2 \mathrm{~h}$ under mild shaking. The supernatant containing released Cbi was collected by 5 seconds centrifugation, at set times and replaced with fresh water or buffer. Concentrations of released Cbi were determined by adding excess potassium cyanide to the solution to convert Cbi to the dicyano form and then by measuring the optical absorbance at $370 \mathrm{~nm}\left(\varepsilon=30000 \mathrm{M}^{-1}\right.$ $\mathrm{cm}^{-1}$ ) according to ref. 36 .

Optical density (absorbance) spectra were recorded on a Thermo Scientific Evolution 201 UV-Visible Double Beam spectrophotometer (Thermo Scientific Inc.) with $1 \mathrm{~cm}$ matched Quartz cells, in a range of wavelength between 250 and $800 \mathrm{~nm}$. Optical density of samples was analysed for one week in order to check stability of the loaded drug. This monitoring period is more than enough for DDS applications. ${ }^{49}$

\section{Results and discussion}

\subsection{Structural investigation}

To investigate surface electric charge, $\zeta$-potential of samples was monitored at different $\mathrm{pH}$ for all the samples in PBS (Fig. 1). The results show pSi-COOH particles having a negative $\zeta$ potential, with a value of $-26.2 \mathrm{mV}$, at physiological $\mathrm{pH}$ (i.e. 7), due to the presence of carboxyl groups onto the surface. Addition of diamine and obtainment of $\mathrm{pSi}-\mathrm{NH}_{2}$, leads to have positive surface charge at physiological $\mathrm{pH}$ without significant effect on the morphology of the samples. ${ }^{12}$ Moreover, $\zeta$-potential variation of free Cbi in PBS as a function of $\mathrm{pH}$ is similar to the one of $\mathrm{pSi}-\mathrm{NH}_{2}$, which could be related to the amine functions of both samples.

In order to allow structural stability and optical properties preservation in water and/or biological liquids, various surface coatings were considered and studied. Firstly, the surface modification with PEG through covalent attachment was

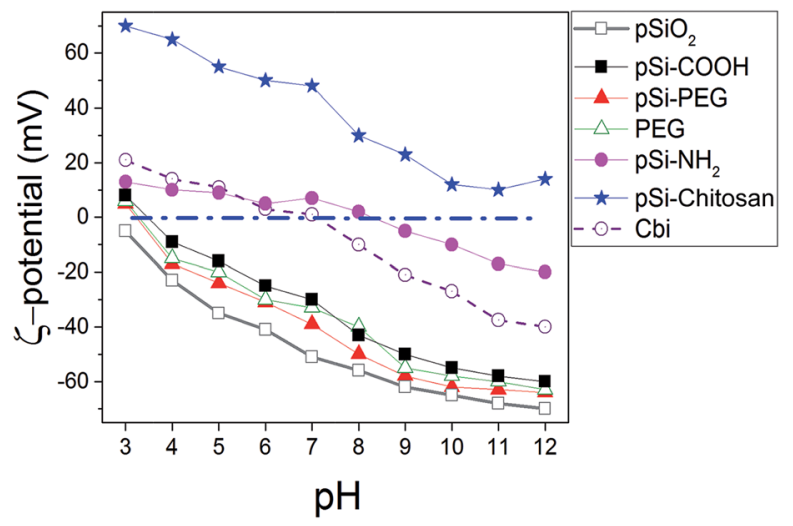

Fig. 1 Variation of $\zeta$-potential as a function of the $\mathrm{pH}$ for $\mathrm{Cbi}$ and different surface coverage of pSi in PBS. attempted. We selected a PEG functionalized at the two ends with an amino and a carboxyl group $\left(\mathrm{H}_{2} \mathrm{~N}-\mathrm{PEG}-\mathrm{COOH}\right)$ respectively, in order to create an amide bond between the amino group of PEG and the carboxyl group present on the $\mathrm{pSi}$ $\mathrm{COOH}$ surface, thus leaving the same surface termination $(-\mathrm{COOH})$ at the end of PEG chain for further possible conjugations (i.e. DDS targeting). This is reflected on the fact that the highly negative $\zeta$-potential $(-37.9 \mathrm{mV}$ in $\mathrm{pH}$ of 7$)$ is maintained. In case of coating with a double amount of PEG (pSi-PEG2), the results in terms of $\zeta$-potential values were similar to those found for pSi-PEG1 and pure PEG, as previously reported. ${ }^{17}$ In fact, variation of $\zeta$-potential for samples with similar surface functionality $(-\mathrm{COOH})$, which are pSi-COOH, pSi-PEG, and PEG, is in the order of the experimental error. Moreover, according to ref. 50 , the $\mathrm{pSiO}_{2}$ has $\zeta$ value slightly lower than other samples which is consistent with the $\zeta$-potential of silica. ${ }^{51}$

Finally, the surface modification with chitosan biopolymer, through physical adsorption and electrostatic interaction, was also investigated (pSi-chitosan). Indeed chitosan, thanks to its abundance of positively charged amino groups, can electrostatically interact with the negative charged $\mathrm{pSi}-\mathrm{COOH}$, thus keeping protected the particles between its chains, meanwhile leaving free amino groups for further conjugation and modification. ${ }^{52}$ Indeed, $\zeta$-potential analysis showed a highly positive value $+48.3 \mathrm{mV}$, due to the amino groups of chitosan, meaning that an efficient coating of the native particles has been obtained. $\zeta$-Potential changes of chitosan functionalized particles are in line with recent published data. ${ }^{53}$

SEM analysis was performed in order to check particle sizes and to assess their morphology, in particular after the organic coating. Native pSi micro-particles are typically elongated (i.e. rod like shape) with few micron size (Fig. 2a). This is due to the fragmentation of the porous silicon layer, which occurs along the vertically oriented pores. Micro-particles size distribution obtained from optical image analysis is large (few microns for the major axis) as expected on the basis of the fabrication approach. ${ }^{12}$ By surface functionalization to obtain $\mathrm{pSi}-\mathrm{COOH}$ (Fig. 2b) roughness of surface increased based on corrosive chemical reactions. By increasing the magnification of SEM, the diameter of the pores has been observed to be about $30 \mathrm{~nm}$ (Fig. 2c). As it was mentioned before, ${ }^{12}$ there is not significant variation on morphology by addition of diamine to get $\mathrm{pSi}-\mathrm{NH}_{2}$ (Fig. 2d). SEM analysis of pSi-PEG1 showed a homogenous sample with rods like particles clearly visible (Fig. 2e). As for pSi-chitosan sample, SEM analysis revealed, indeed, the presence of microparticles, spheres-like shaped, homogeneously dispersed in the sample (Fig. 2f) with a dimensions of about 2-5 $\mu \mathrm{m}$. In fact, based on the chemical reaction during the functionalization, homogeneity of $\mathrm{pSi}-\mathrm{COOH}$ sample morphology was increased.

To validate the organic functionalization procedure, FTIR analysis was performed, which showed that the expected functional groups were correctly grafted (Fig. 3). For the sake of clarity, all spectra have been normalized and shifted along the $y$ axis. The native pSi particles present the characteristic vibration mode at $2100-2300 \mathrm{~cm}^{-1}$, and also the peak at $790-800 \mathrm{~cm}^{-1}$, which are due to $\mathrm{SiH} .{ }^{54}$ On the contrary, $\mathrm{pSi}-\mathrm{COOH}$ sample did 

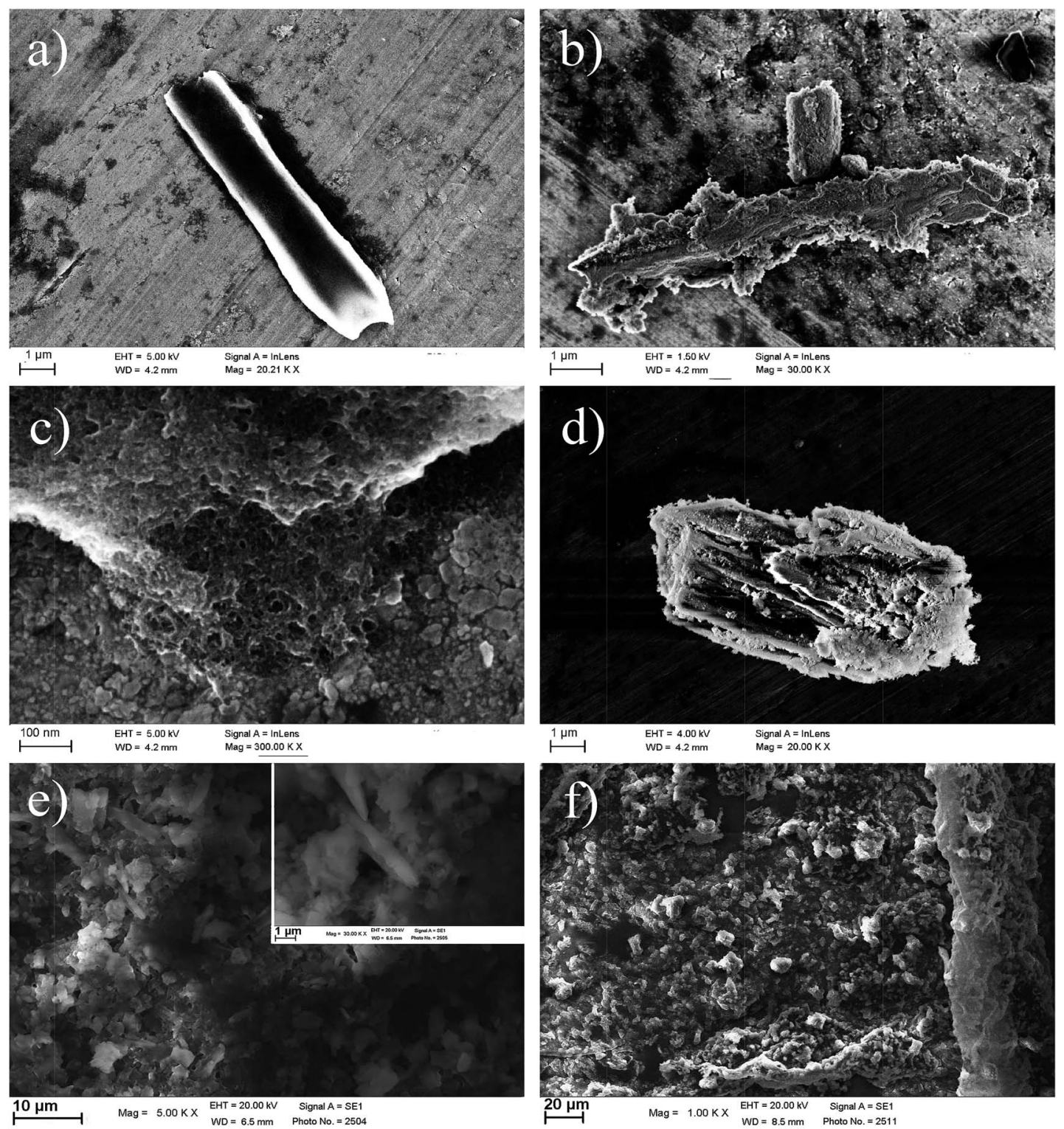

Fig. 2 SEM images of native pSi (a), pSi-COOH sample in one-micron scale to show particle rod-like shape (b) and in nanometre scale to better point out surface porosity (c), pSi- $\mathrm{NH}_{2}$ sample (d), pSi-PEG1 sample in 10-micron scale (e) with a higher magnification in the inset, and pSichitosan sample in 20-micron scale (f).

not present any characteristic $\mathrm{SiH}$ vibration mode at 2100-2300 $\mathrm{cm}^{-1}$, but a strong peak at $1060-1110 \mathrm{~cm}^{-1}$ due to the presence of asymmetric stretching of $\mathrm{Si}-\mathrm{O}-\mathrm{Si}$ bond.$^{55}$ Interestingly, also a signal at $1715 \mathrm{~cm}^{-1}$ appears, which is the typical stretching vibration of $-\mathrm{C}=\mathrm{O}$ containing compounds thus confirming the presence of $\mathrm{COOH}$ groups onto the surface. ${ }^{57}$ The other peak at $873 \mathrm{~cm}^{-1}$ is related to $\mathrm{Si}-\mathrm{O}$ bending.

In the case of PEG functionalization, it is possible to observe a strong peak at $1046 \mathrm{~cm}^{-1}$ as typical stretching vibration in ethers, the presence of the amide bond vibrations of $-\mathrm{C}=\mathrm{O}$ at $1644 \mathrm{~cm}^{-1}$ (amide I band) and of -NH stretching at 3200-3400 $\mathrm{cm}^{-1}$ which overlaps with $\mathrm{Si}-\mathrm{OH}$ peak. ${ }^{56}$ After the coating of pSi-COOH samples with chitosan, the peak around $1070 \mathrm{~cm}^{-1}$ was retained and a new band is found at $1550-1600 \mathrm{~cm}^{-1}$ due to the presence of primary amides, which are abundant in chitosan. Also Si-OH vibrations appeared at $3200-3600 \mathrm{~cm}^{-1}$ as a broad band, which is typical of $-\mathrm{OH}$ modes, while alkyl $-\mathrm{CH}$ stretching modes appeared at $2850-2950 \mathrm{~cm}^{-1} \cdot{ }^{57}$

\subsection{Emission stability in biological media}

Fig. 4 reports PL spectra of all the samples excited at $350 \mathrm{~nm}$. Based on $\mathrm{pSi}-\mathrm{COOH}$ and $\mathrm{pSi}-\mathrm{NH}_{2}$ have more compatibility in ethanol, PL of these samples was acquired in ethanol where functionalized $\mathrm{pSi}$ has been shown to be stable for years. ${ }^{21}$ Conversely, for the other samples PL spectra were obtained in PBS, because PEG and chitosan have high affinity for aqueous environments. PL of all samples was normalized by comparison with $\mathrm{pSi}-\mathrm{COOH}$, as reference (see Table 2). As it can be seen, $\mathrm{pSi}-\mathrm{COOH}$ has a peak in the red region (about $665 \mathrm{~nm}){ }^{58}$ It is 


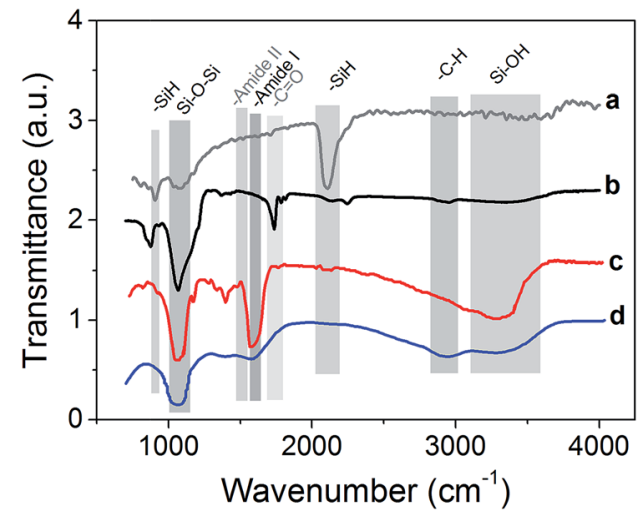

Fig. 3 FTIR measurements of the pSi microparticles before and after surface functionalization and successive coating: native psi (a), pSi-COOH functionalized (b) and surface modified by PEG (c) and chitosan (d).

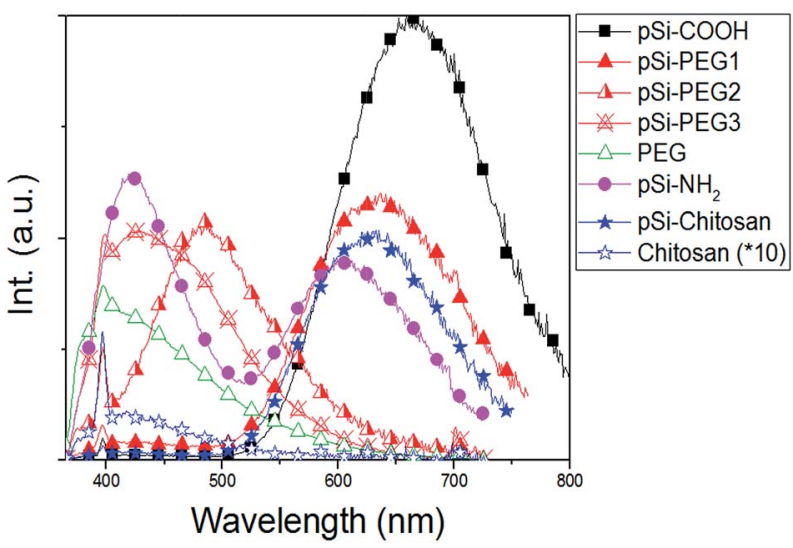

Fig. $4 \mathrm{PL}$ spectra of samples in Table 1 (excitation at $350 \mathrm{~nm}$ ). PL curves normalized based on $\Phi$ value from Table 2 . Chitosan $(\times 10)$ signal is reported for comparison with $\mathrm{pSi}$-chitosan.

Table 2 Optical quantum yields obtained by comparative method

\begin{tabular}{|c|c|c|c|}
\hline Sample & $\Phi(\%)$ & $\lambda_{(\max )}(\mathrm{nm})$ & FWHM (nm) \\
\hline $\mathrm{pSi}-\mathrm{COOH}$ & 1.7 & 660 & 154 \\
\hline $\mathrm{pSi}-\mathrm{NH}_{2}$ red band & 0.7 & 603 & 140 \\
\hline pSi- $\mathrm{NH}_{2}$ blue band & 0.6 & 422 & 88 \\
\hline pSi-PEG1 & 1.1 & 636 & 153 \\
\hline pSi-PEG2 & 0.9 & 485 & 115 \\
\hline pSi-PEG3 & 0.8 & 430 & 131 \\
\hline PEG & 0.5 & 410 & 110 \\
\hline pSi-chitosan & 0.8 & 635 & 139 \\
\hline Chitosan & 0.02 & 419 & 105 \\
\hline
\end{tabular}

worth noting that the sharp peak at about $390 \mathrm{~nm}$ is due to the Raman effect of water. ${ }^{59}$ After functionalization and further suspension in PBS, the pSi-PEG and the pSi-chitosan PL bands are very similar in shape, presenting just a blue-shift with respect to $\mathrm{pSi}-\mathrm{COOH}$ sample. The blue-shift of the red band is consistent with quantum effects. ${ }^{60}$ Chitosan, alone, has a low emission and, as it can be seen from Fig. 4, there is no correlation between PL of chitosan and pSi-chitosan spectra. ${ }^{61}$ It must be noticed that $\mathrm{pSiO}_{2}$ has no optical emission band in the range of interest. ${ }^{37}$

PL of pSi-PEG2 and pSi-PEG3 has been shifted more, about $180 \mathrm{~nm}$ and $235 \mathrm{~nm}$, respectively. It must be considered that even though the microparticles functionalized with both PEG concentrations present similar molecular structure and size, the larger amount of PEG (i.e.: $\mathrm{HCl} \cdot \mathrm{H}_{2} \mathrm{~N}-\mathrm{PEG}-\mathrm{COOH}$ ) used during functionalization probably leads to a stronger surface modification. The correlation between the spectral shift and the concentration of the reactant (NH-PEG-COOH) suggests that some modification of the light emitting silicon structures occurs during the functionalization, probably due to silicon oxidation catalysed by the amino group, as already reported for other amines. ${ }^{40}$ For the sake of clarity, PL of pure PEG has been measured: as it can be seen, PEG, itself, has a significant emission band in the blue region $(400-450 \mathrm{~nm}){ }^{62}$

The emission quantum yield $(\Phi)$ of all samples dispersed in PBS has been estimated by comparative method (Table 2). The obtained values for pSi samples (between $0.8 \%$ and $1.1 \%$ ) are not significantly reduced if compared to that of the $\mathrm{pSi}-\mathrm{COOH}$ that is about $1.7 \%$. On the other hand, pure chitosan has negligible light emission efficiency.

Unlike the blue shift, the $\Phi$ reduction is not the same after PEG and chitosan functionalization. As for the pSi-PEG1 sample, $\Phi$ is reduced of about $33 \%$, as previously reported. ${ }^{18} \mathrm{~A}$ similar decrease of the quantum yield is observed for $\mathrm{pSi}-\mathrm{NH}_{2}$. However, quantum yield is further reduced by increasing PEG amount (down to 0.9 and 0.8 for the pSi-PEG2 and pSi-PEG3 samples, respectively). As for pSi-chitosan sample, the quantum yield is about 0.8 , which is roughly half of $\mathrm{pSi}-\mathrm{COOH}$ sample and the same as for pSi-PEG3. The reduction with respect to PSi-PEG1 sample can be attributed to the fact that the chitosan matrix acts as higher optical absorber especially in the blue range. ${ }^{63}$ In fact, it appears more opaque due to the larger passivation upon the pSi-COOH structures. These quantum yield reductions by surface functionalization are in line with recent experimental data of pSi by others. ${ }^{64}$

Until now, we obtained a long-lasting PL stabilization in organic solvents (toluene and ethanol) for years, ${ }^{12}$ but the PL half-life in aqueous environment remained of the order of few hours. The change over time of the PL spectral features of pSi samples in PBS have been studied and compared in Fig. 5. This figure shows variation of the red emission band for all the tested samples (solid lines), and variation of the second band in the blue region only for pSi-chitosan and $\mathrm{pSi}-\mathrm{NH}_{2}$ samples (dash lines). The intensities have been normalized by eqn (2), in order to let them be appreciable in the same figure.

$$
I=\left(\frac{I_{\mathrm{d}}}{I_{\mathrm{i}}}\right)_{\text {sample }} \times\left(\frac{\Phi_{\text {sample }}}{\Phi_{\mathrm{pSi}-\mathrm{COOH}}}\right)
$$

herein, $I_{\mathrm{i}}$ and $I_{\mathrm{d}}$ are the PL intensities of the sample in PBS immediately after suspension and after "d" days. $\Phi_{\text {sample }}$ and $\Phi_{\mathrm{pSi}-\mathrm{COOH}}$ are the quantum yield of the sample and $\mathrm{pSi}-\mathrm{COOH}$, respectively. Indeed, the initial point (at time zero) is just related to reduction of $\Phi$ value due to functionalization in comparison with pSi-COOH. 


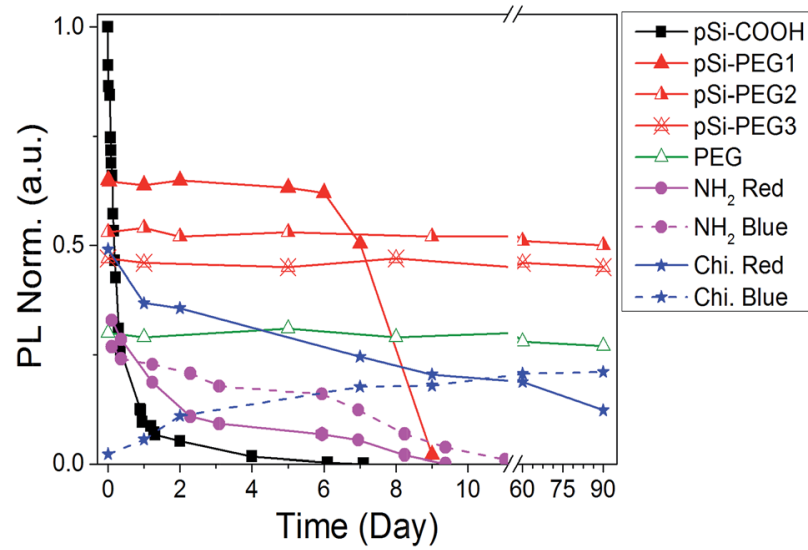

Fig. $5 \mathrm{PL}$ intensity vs. time (day) in PBS buffer. Blue band of $\mathrm{pSi}-\mathrm{NH}_{2}$ and $\mathrm{pSi}$-chitosan are indicated by dash lines.

The half-life of the pSi-COOH in PBS is about $5 \mathrm{~h}$, which is longer than what reported by other authors (about $3 \mathrm{~h}$ ). ${ }^{65}$ The half-life increases to more than 7 days after suspension in PBS for the pSi-PEG1, without any blue shift. It is worth noting that one-week optical stability is more than what is generally required for bioimaging applications and it is also promising for photo-thermal therapy. ${ }^{66}$

By increasing the amount of PEG (pSi-PEG2 and pSi-PEG3), $\Phi$ reduces of about $30 \%$ and PL peak shifts up to about $200 \mathrm{~nm}$ from red to blue spectral region (Table 2). After this initial variation, the PL band is stable up to three months (90 days). To the best of our knowledge, this stability of the visible light emission (in both intensity and shape) in PBS for Si-based nano or micro-structures has not been reported till now. Moreover, these data may indicate that the PL band could be further tuned by varying the PEG amount thus having an additional postprocessing tool for wavelength shifting without influencing the intensity stability over the time.

Similar behaviour was observed for pSi-chitosan showing a long-term optical stability in PBS (Fig. S3†): the red band (at about $635 \mathrm{~nm}$ ) decreases with time (days), while the blue one (at about $400 \mathrm{~nm}$ ) appears and increases with time (days). The decrease of the red emission (about 30\% of the initial value after 15 days) can be due to the slow but progressive oxidation of luminescent silicon microparticles ${ }^{67}$ that reduces the dimensions of the nanocrystallites and introduces oxygen defects. ${ }^{68}$ For this reason, the red emission (related to quantum confinement) progressively decreases in intensity and slightly shifts to shorter wavelength (from about $635 \mathrm{~nm}$ to about $600 \mathrm{~nm}$ after 90 days). Conversely, the optical emission at $400 \mathrm{~nm}$ (blue band) has significantly more emission thus suggesting that it might be attributed to the surface defects at the nanocrystallites induced by nitrogen containing compounds ascribed to the amino groups, ${ }^{69}$ which are progressively released because of the dissolution of chitosan and interaction with $\mathrm{pSi}^{.70,71}$

According to the above results, we were able to stabilize the PL intensity and feature it up to months in a high ionic strength aqueous buffer such as PBS. To the best of our knowledge, this is the first functionalization protocol that stabilizes visible PL of functionalized pSi for such a long period: 90 days.

\subsection{Interaction of pSi with Cbi}

As previously said, the surface chemistry of pSi plays a fundamental role in the adsorption of drugs within the porous matrix. In order to understand the mechanisms regulating such an interaction, we first exploit optical (UV-Visible) absorption to monitor the absorbance spectrum of Cbi. The spectra of the cobinamide solution after interaction with pSi for $3 \mathrm{~h}$ revealed significant changes compared to that of the reference cobinamide solution (Fig. 6).

The characteristic optical absorption peaks of cobinamide are at about 500 and $525 \mathrm{~nm}$ (cobalt is in $\mathrm{Co}^{3+}$ state). ${ }^{45}$ After $3 \mathrm{~h}$ (big dot line of Fig. 6), the peaks at about 500 and $525 \mathrm{~nm}$ disappeared, while a large absorption peak centred at $430 \mathrm{~nm}$ raises according to the reduction of $\mathrm{Co}^{3+}$ centre to $\mathrm{Co}^{2+}$ by interaction with the Si surface. After longer interaction $(24 \mathrm{~h})$ it occurs also a complete loss of the cobalt-corrin ring coordination as clearly shown by the disappearance of this peak, that means that Cbi is completely decomposed. ${ }^{46}$ On the other hand, our results showed that $\mathrm{Cbi}$ is stable when in contact with $\mathrm{pSiO}_{2}$ in PBS solution (see dots in Fig. 6) in agreement with previous findings. ${ }^{36}$

Therefore, we experimentally investigated which different surface functionalization is efficient to avoid alteration (e.g. reducing process) of the loaded Cbi. Table 3 shows the reaction times of Cbi reduction and its decomposition as a function of the different functionalized pSi samples. In this table, the column "step 1" shows the time measured to get the maximum intensity of the $430 \mathrm{~nm}$ peak (i.e. $\mathrm{Co}^{3+} \rightarrow \mathrm{Co}^{2+}$ transformation) while "step 2" the time of complete Cbi decomposition associated to the disappearing of it.

The time courses of Cbi reduction experimentally recorded can be attributed to the surface charge: samples with negative charge (pSi-COOH, pSi-PEGs) have low or no effect on Cbi modification. In fact, carboxyl functionalization significantly reduces the reaction rates of about a factor 3 with respect to native pSi for both steps: $\mathrm{Co}^{3+}$ reduction in $10 \mathrm{~h}$ instead of $3 \mathrm{~h}$

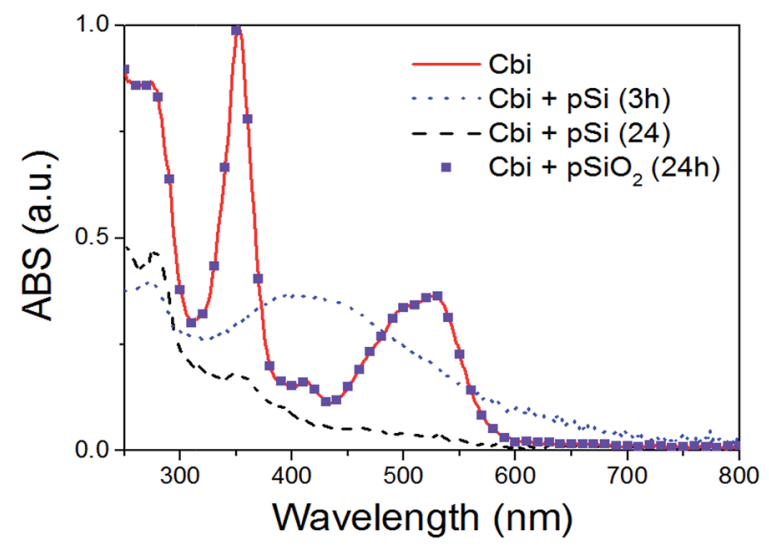

Fig. 6 Optical absorption of $\mathrm{Cbi}$ after the interaction with $\mathrm{pSi}$, or $\mathrm{pSiO}_{2}$, in PBS as a function of time. 
Table 3 Observed times for reaction of Cbi with pSi samples. Step 1 corresponds to the reduction $\mathrm{Co}^{3+} \rightarrow \mathrm{Co}^{2+}$, step 2 is when complete decomposition of Cbi occurs

\begin{tabular}{lll}
\hline Samples & Step 1 & Step 2 \\
\hline $\mathrm{pSi}$ & $3 \mathrm{~h}$ & $24 \mathrm{~h}$ \\
$\mathrm{pSiO}_{2}$ & $\mathrm{NO}^{a}$ & $\mathrm{NO}$ \\
$\mathrm{pSi}-\mathrm{COOH}$ & $10 \mathrm{~h}$ & 3 days \\
$\mathrm{pSi}-\mathrm{PEGs}$ & $\mathrm{NO}$ & $\mathrm{NO}$ \\
$\mathrm{pSi}-\mathrm{NH}_{2}$ & $\sim$ Few s & $1 \mathrm{~h}$ \\
pSi-chitosan & $\sim$ Few s & $1 \mathrm{~h}$
\end{tabular}

${ }^{a}$ No reaction carried out during one week. ${ }^{b}$ In the order of few seconds.

and Cbi complete decomposition 3 days instead of 1 day. More attractively, we report here that the PEG functionalization has no effect on the structure of Cbi, similarly to what already reported for completely oxidized pSi. It is worth noting that no optical absorption modification was observed after one week for both $\mathrm{pSiO}_{2}$ and PEG functionalized pSi samples.

On the other hand, samples with positive surface charge (pSi- $\mathrm{NH}_{2}$ and $\mathrm{pSi}$-chitosan) showed very fast degradation (in the order of few seconds for step 1 and about 1 hour for step 2) of the Cbi. The fast reaction (few seconds) of Cbi with samples containing amine functional groups could be related to the reduction of Cbi by nitrogen impurities as previously reported. ${ }^{\mathbf{4 4}}$ In fact, based on this report, kinetic rate of this reduction is in the order of seconds.

After the chemical stability assessment of the Cbi drug once incorporated into the silicon porous matrix, the other fundamental features to be tested for a reliable DDS are (i) the loading capacity and efficiency and (ii) the release time profile. Cbi loading efficiency was measured by extracting the loaded cobinamide from the pSi microparticle matrix by a treatment with a sodium hydroxide solution ( $\mathrm{pH}$ 9), that dissolved the $\mathrm{Si}$ and $\mathrm{SiO}_{2}$ components thus releasing the drug payload into the solution. We quantified the Cbi amount by converting it into the dicyano form (by adding excess potassium cyanide) and by measuring the optical absorbance at $370 \mathrm{~nm} .^{36}$

The results are reported in Table 4 . The cobinamide loadings were 110 and $32 \mu \mathrm{g}$ of Cbi per mg of microparticle, in average, for the $\mathrm{pSi}$ and $\mathrm{pSiO}_{2}$, respectively. The loading capacity

Table 4 Cbi loading capacity for pSi with different surface chemistry

\begin{tabular}{lcc}
\hline Sample & $\begin{array}{l}\text { Loading capacity } \\
(\mu \mathrm{g} \text { of Cbi per } \mathrm{mg} \text { of } \mathrm{pSi})\end{array}$ & $\begin{array}{l}\zeta \text {-Potential }^{a} \\
(\mathrm{mV})\end{array}$ \\
\hline Native pSi & $110 \pm 15$ & -14 \\
$\mathrm{pSiO}_{2}$ & $32 \pm 2$ & -56 \\
pSi-COOH & $380 \pm 32$ & -25 \\
pSi-PEG1 & $225 \pm 18$ & -31 \\
pSi-PEG2 & $120 \pm 10$ & -33 \\
pSi-PEG3 & $176 \pm 14$ & -32 \\
PEG & $52 \pm 9$ & -30 \\
pSi-NH & $12 \pm 2$ & 2 \\
pSi-chitosan & $18 \pm 1$ & 30
\end{tabular}

${ }^{a}$ Measured at pH 6, which is used for loading Cbi. dramatically reduced by silicon oxidation because of the reduction of available surface area. This fact strongly limits the use of oxidized pSi for DDS. Loading capacity for pSi-PEG and $\mathrm{pSi}-\mathrm{COOH}$ is about 225 and $380 \mu \mathrm{g}$ of Cbi per mg of pSi, respectively. Indeed, the loading capacity increased significantly with respect to oxidized pSi ( 2 and 5 fold, respectively). The loading capacity for pSi-PEG2 is in the same range as that of native pSi. Functionalized PEG samples show less capacity with respect to carboxyl ones probably due to the longer chains of PEG, which lead to a reduction of the pores volume available for the Cbi loading. This is confirmed by the fact that with increasing PEG amount (see pSi-PEG2) the pores volume is less and less available to carry the drug. This effect can be however mitigated by an increase of the pores size in the native pSi by playing with the electrochemical etching parameters in the fabrication of the porous silicon. ${ }^{72}$

It is worth noting that the large loading capacity (attributed to electrostatic interaction) here measured in $\mathrm{pSi}-\mathrm{COOH}$ sample (about $40 \%$ ), has not ever seen even by the oxidation trapping $^{36}$ and other methodologies. ${ }^{50}$ However, as shown above, pSi-COOH has not good optical stability in PBS in comparison with samples functionalized with PEG. Therefore, pSi-PEG with a loading capacity up to $225 \mu \mathrm{g}$ of Cbi per mg of microparticle is the best candidate for a reliable DDS.

On the other hand, positive surface charge leads to a poor loading capacity with respect to native $\mathrm{pSi}$, and even less than the oxidized one $\left(\mathrm{pSiO}_{2}\right)$ : about 12 and $18 \mu \mathrm{g}$ Cbi per mg of microparticles for $\mathrm{pSi}-\mathrm{NH}_{2}$ and $\mathrm{pSi}$-chitosan samples, respectively.

As a further and last step of this experimental study, we verified the release of Cbi from pSi. Results are summarized in Fig. 7, where the Cbi release is monitored as a function of time (days). As it is obvious, when there is not attraction between pSi and $\mathrm{Cbi}$, Cbi will release from pSi easily. According to this fact and Table 4, we can categorize the Cbi release into two groups, respectively faster and slower if compared to a medium release rate represented by native $\mathrm{pSi}$ and $\mathrm{pSiO}_{2}$ samples. As it can be clearly observed in Fig. 7, we found faster release rate for the positively charged samples and slower rate for the negatively charged ones (refer to the $\zeta$-potential values in the Table 4).

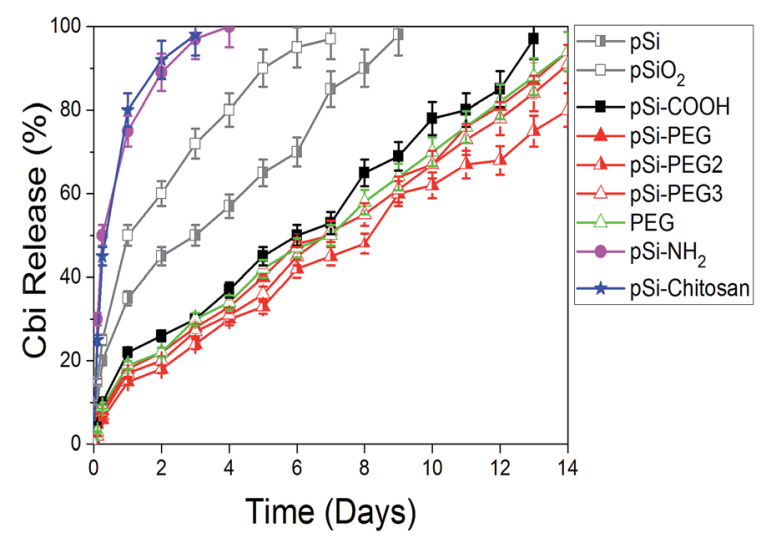

Fig. 7 First two-weeks release profile of Cbi into PBS from pSi samples as a function of the surface coating 
In the first case, $\mathrm{pSi}-\mathrm{NH}_{2}$ and $\mathrm{pSi}$-chitosan samples show about $50 \%$ of the drug released within few hours and more than $95 \%$ within 3 days. This fast release might be attributed to the electrostatic repulsion of similar surface charge. Release rate from chitosan and amine functionalized samples are quite the same, in opposite of having different $\zeta$-potentials. The expected faster release rate of $\mathrm{Cbi}$ in the pSi-chitosan sample is probably contrasted by the fact that the long polymeric chains of chitosan can act as traps for the Cbi molecules.

In the second group associated to the negatively charged samples, the Cbi release is quite linear with time with a slope of about $6 \%$ release per day. Rate of pSi-PEG and pSi-PEG2 is slightly lower than that of $\mathrm{pSi}-\mathrm{COOH}$, which could be related to the trapping of Cbi in the longer chains of PEG samples.

Moreover, the release rate by native $\mathrm{pSi}$ is lower based on trapping of Cbi during oxidation of pSi. Indeed, surface oxidation of pSi in PBS leads to closing of porosity due to oxygen absorption. ${ }^{37}$ This phenomenon cases trapping of Cbi. ${ }^{19}$ Then release of Cbi needs first this oxidized layer to dissolve in PBS, which is time consuming.

Cbi has no PL properties in the visible range. Then, PL of loaded samples is just based on pSi. Additionally, native pSi and $\mathrm{pSiO}_{2}$ samples should be avoided since $\mathrm{pSiO}_{2}$ does not have PL properties, and native pSi quenched during $3 \mathrm{~h}$ after the loading of Cbi. Also, PL properties of Cbi loaded pSi- $\mathrm{NH}_{2}$ and pSi-chitosan samples were similar with unloaded ones, which it could be related to negligible amount of loading (less than $2 \%$ ).

As previously showed in the Fig. 5, pSi-COOH sample presents a dramatic quenching in PBS. However, the loading of Cbi leads to significantly mitigate the optical quenching rate: Fig. S4a † shows about a factor 2 of improvement in time (days). This improvement of the light emission stability is attributed to the facts that Cbi works as a barrier for surface oxidation of silicon. On the other hand, Fig. S4b $\dagger$ demonstrates that Cbi does not have any effects, as expected, on PL band of the pSi$\mathrm{COOH}$ sample thus preserving the optical properties (in peak maximum wavelength and shape).

As for PEG functionalized samples, we did not observe any variation of the optical properties as a function of Cbi loading. The reason is attributed to the electrostatically attraction of $\mathrm{Cbi}$ with PEG, without any interaction with the silicon core structure. In fact, the pSi-PEG was already stable from the optical point of view right in reason of a better passivation of the silicon surface.

All these results are in favour of the idea that a reliable delivery drug system should consist of a carrier that does not damage either the drug but maintain or even improve the optical properties of the carrier itself thus paving the way to an intrinsic (without the need of additional fluorophores) combined imaging.

\section{Conclusions}

In this experimental study, luminescent porous silicon (pSi) microparticles have been obtained based on anodization method. In order to reach structural and optical stabilization in biological media (such as water or PBS), different surface functionalization procedures were investigated. Attachment of the different functional groups onto the pSi surface were confirmed by FTIR analysis. Among them, PEG and chitosan were the more effective polymer for pSi stabilization, but with opposite electric surface charge as determined by $\zeta$-potential measurements.

Visible light emission of pSi stabilized for over three months has been reported for the first time for both PEG and chitosan coating of pSi microparticles. In fact, it is very suitable for biomedical applications to offer orthogonal potential functionalization since they bear in the outer shell carboxylic and amino functional groups, negatively and positively charged, respectively.

In fact, in addition to the poor stability in biological media, the concern about pSi in biomedicine is the reducing activity of pSi itself that degrades the drug. To test the improved performances of polymer coated pSi as DDS, Cbi was selected as a reference drug based on its high sensitivity to reduction. Experimental data demonstrated that PEG functionalization leads to avoid reduction and decomposition of $\mathrm{Cbi}$; in addition, it leads to improve the loading amount and the release profile. Moreover, Cbi improves PL stability.

In conclusion, this experimental study shows that $\mathrm{pSi}$ microparticles, opportunely functionalized, are a reliable and promising candidate for theranostics due to the high drug loading capacity, the possibility of controlling fast or long release rate accordingly, no drug deterioration, and to the longterm optical stability in biological liquids.

\section{Acknowledgements}

This work has been done within the Nanomedicine Initiative funded by Fondazione Cariverona (Verona). University of Bologna is also gratefully acknowledged. We thank Prof. Antonella Motta and Lorenzo Moschini, Department of Material Engineering, University of Trento for acquisition of SEM images. We are grateful to Dr Martino Bianconi for his technical assistance in HPLC measurements.

\section{References}

1 L. T. Canham, Adv. Mater., 1995, 7, 1033.

2 J. Salonen, A. M. Kaukonen, J. Hirvonen and P. V. Lehto, J. Pharm. Sci., 2008, 97, 632.

3 Q. Fang, J. Wang, S. Gu and R. B. Kaspar, J. Am. Chem. Soc., 2015, 137(26), 8352.

4 O. Bisi, S. Ossicini and L. Pavesi, Surf. Sci. Rep., 2000, 38, 1.

5 M. L. Sailor, Chemical reactivity and surface chemistry of porous silicon, in Handbook of Porous silicon, Springer International Publishing, Switzerland, 2014, p. 335.

6 S. P. Low and N. H. Voelcker, Biodegradability of porous silicon, in Handbook of Porous silicon, Springer International Publishing, Switzerland, 2014, p. 381.

7 C. F. Wang, E. M. Mäkilä, M. H. Kaasalainen and D. Liu, Biomaterials, 2014, 35(4), 1257.

8 C. F. Wang, M. P. Sarparanta, E. M. Mäkilä and M. L. K. Hyvönen, Biomaterials, 2015, 48, 108. 
9 H. Hou, A. Nieto, F. Ma, W. R. Freeman and M. J. Sailor, J. Controlled Release, 2014, 178, 46.

10 E. J. Anglin, L. Cheng, W. R. Freeman and M. J. Sailor, Adv. Drug Delivery, 2008, 60, 1266.

11 R. J. Martín-Palma, J. Hernández-Montelongo, V. TorresCosta, M. Manso-Silván and Á. Muñoz-Noval, Expert Opin. Drug Delivery, 2014, 11, 1273.

12 N. Daldosso, A. Ghafarinazari, P. Cortelletti, L. Marongiu, M. Donini, V. Paterlini, P. Bettotti, R. Guider, E. Froner, S. Dusi and M. Scarpa, J. Mater. Chem. B, 2014, 2, 6345.

13 J. H. Park, L. Gu, G. von Maltzahn, E. Ruoslahti, S. N. Bhatia and M. J. Sailor, Nat. Mater., 2009, 8(4), 331.

14 M. A. Avidad, A. Nieto, A. Salinas-Castillo, L. Capitan-Vallvey, G. Miskelly and M. J. Sailor, Nano Res. Lett., 2014, 9(1), 1.

15 G. M. Lanza, Nat. Nanotechnol., 2015, 10, 301.

16 S. Näkki, J. Rytkönen, T. Nissinen, C. Florea and J. Riikonen, Acta Biomater., 2015, 13, 207.

17 B. Godin, J. Gu, R. E. Serda, R. Bhavane, E. Tasciotti, C. Chiapinni, X. Lu, T. Tanaka, P. Decuzzi and M. Ferrari, J. Biomed. Mater. Res., Part A, 2010, 94(4), 1236.

18 R. R. Chandler-Henderson, B. Sweryda-Krawiec and J. L. Coffer, J. Phys. Chem., 1995, 99, 8851.

19 E. C. Wu, J. S. Andrew, A. Buyanin, J. M. Kinsella and M. J. Sailor, Biomaterials, 2011, 32, 1957.

20 E. Secret, M. Maynadier, A. Gallud and A. Chaix, Adv. Mater., 2014, 26(45), 7643.

21 A. Ghafarinazari, V. Paterlini, P. Cortelletti, P. Bettotti, M. Scarpa and N. Daldosso, J. Nanosci. Nanotechnol., 2017, 17(2), 1240.

22 N. K. Hon, Z. Shaposhnik, E. D. Diebold, F. Tamanoi and B. Jalali, ECS Trans., 2013, 45, 7.

23 J. Joo, J. F. Cruz, S. Vijayakumar, J. Grondek and M. J. Sailor, Adv. Funct. Mater., 2014, 24, 5688.

24 K. H. Wu, C. W. Li and J. H. Liu, Microelectron. Eng., 2015, 148, 70.

25 Y. H. Ogata, T. Tsuboi, T. Sakka and S. Naito, J. Porous Mater., 2000, 7, 63.

26 E. Locatelli, I. Monaco and M. Comes Franchini, RSC Adv., 2015, 5, 21681.

27 B. C. Mei, K. Susumu, I. L. Medintz and H. Mattoussi, Natur. Prot., 2009, 4(3), 412.

28 K. Knop, R. Hoogenboom, D. Fischer and U. S. Schubert, Angew. Chem., Int. Ed., 2010, 49, 6288.

29 S. K. Nitta and K. Numata, Int. J. Mole. Scien., 2013, 14, 1629.

30 M. N. V. R. Kumar, Reac. Func. Poly., 2000, 46, 1.

31 A. M. Piras, S. Sandreschi, G. Maisetta and S. Esin, Pharma. Res., 2015, 32(7), 2259.

32 E. C. Wu, J. S. Andrew, A. Buyanin, J. M. Kinsella and M. J. Sailor, Chem. Commun., 2011, 47(20), 5699.

33 K. L. Jarvis, T. J. Barnes and C. A. Prestidge, Langmuir, 2010, 26, 14316.

34 S. Low, L. Canham and N. Voelcker, Bio Mater., 2006, 27(26), 4538.

35 CRC Handbook of Chemistry and Physics, ed. R. David, CRC Press, Boca Raton, FL, 87th edn, 2006, ISBN 0-8493-0487-3.

36 N. L. Fry, G. R. Boss and M. J. Sailor, Chem. Mater., 2014, 26, 2758.
37 A. Ghafarinazari, E. Zera, A. Lion, M. Scarpa, G. D. Soraru and N. Daldosso, Thermo. Acta, 2016, 623, 6.

38 G. Aggarwal, P. Mishra, B. Joshi and S. S. Islam, J. Porous Mater., 2014, 21, 23.

39 F. Peng, Y. Su, Y. Zhong, C. Fan and S. T. Lee, Acc. Chem. Res., 2014, 47(2), 612.

40 E. Froner, R. Adamo, Z. Gaburro, B. Margesin, L. Pavesi, E. Rigo and M. Scarpa, J. Nanopart. Res., 2006, 8(6), 1071.

41 K. E. Broderick, V. Singh, S. Zhuang, A. Kambo, J. C. Chen, V. S. Sharma, R. B. Pilz and G. R. Boss, J. Biol. Chem., 2005, 208(10), 8678.

42 K. Proinsias, M. Giedyk and D. Gryko, Chem. Soc. Rev., 2013, 42, 6605.

43 V. S. Sharma, R. B. Pilz, G. R. Boss and D. Magde, Biochemistry, 2003, 42(29), 8900.

44 S. H. Ford, A. Nichols and M. Shambee, J. Inorg. Biochem., 1991, 41, 235.

45 D. Timmerman, J. Valenta, K. Dohnalová, W. De Boer and T. Gregorkiewicz, Nat. Nanotechnol., 2011, 6(11), 710.

46 A. Ogunsipe, J.-Y. Chen and T. Nyokong, New J. Chem., 2004, 28(7), 822.

47 J. H. Ahire, Q. Wang, P. R. Coxon, G. Malhotra, R. Brydson, R. Chen and Y. Chao, ACS Appl. Mater. Interfaces, 2012, 4, 3285 .

48 T. Tanaka, L. S. Mangala, P. E. Vivas-Mejia, R. Nieves-Alicea, A. P. Mann, E. Mora, H. D. Han, M. K. Shahzad, X. Liu and R. Bhavane, Cancer Res., 2010, 70(9), 3687.

49 J. Salonen, Drug delivery with porous silicon, in Handbook of Porous silicon, Springer International Publishing, Switzerland, 2014, p. 909.

50 M. Kaasalainen, E. Mäkilä, J. Riikonen, M. Kovalainen, K. Järvinen, K. H. Herzig, V. P. Lehto and J. Salonen, Int. J. Pharm., 2012, 431, 230.

$51 \mathrm{~J} . \quad$ M. Rosenholm, T. Czuryszkiewicz, F. Kleitz, J. B. Rosenholm and M. Lindén, Langmuir, 2007, 23, 4315.

52 N. Shrestha, M. A. Shahbazi, F. Araújo, H. Zhang, E. M. Mäkilä, J. Kauppila, B. Sarmento, J. J. Salonen, J. T. Hirvonen and H. A. Santos, Biomaterials, 2014, 35(25), 7172.

53 S. H. Chang, H. T. Victor Lin, G. J. Wu and G. Jane Tsai, Carbohydr. Polym., 2015, 134, 74.

54 Y. Ogata, H. Niki, T. Sakka and M. Iwasaki, J. Electrochem. Soc., 1995, 142, 195.

55 G. Korotcenkov and B. K. Cho, Crit. Rev. Solid State Mater. Sci., 2010, 35(3), 153.

56 C. T. Johnston and Y. O. Aochi, Methods of soil analysis. Part 3-chemical methods, 1996, p. 269.

$57 \mathrm{~J}$. Coates, Interpretation of infrared spectra, a practical approach, in Encyclopedia of analytical chemistry, 2000.

58 G. B. Li, L. S. Liao, X. B. Liu, X.-Y. Hou and X. Wang, Appl. Phys. Lett., 1997, 70, 1284.

59 I. R. Rao, Nature, 1930, 125, 600.

60 J. Rytkönen, R. Miettinen, M. Kaasalainen, V.-P. Lehto, J. Salonen and A. Närvänen, J. Nanomater., 2012, 896562.

61 H. Huang, F. Liu, S. Chen, Q. Zhao, B. Liao, Y. Long, Y. Zeng and X. Xia, Biosens. Bioelectron., 2013, 42, 539. 
$62 \mathrm{H} . \mathrm{Xu}, \mathrm{T}$. Zhu and R. P. Yu, Study on the absorption and fluorescence spectra of ethylene glycol and glycerol, Guangpuxue Yu Guangpu Fenxi, 2007, 27(7), 1381.

63 D. E. Azofeifa, H. J. Arguedas and W. E. Vargas, Opt. Mater., 2012, 35(2), 175.

64 K. Dohnalová, A. N. Poddubny and A. A. Prokofiev, Light: Sci. Appl., 2013, 2, 47.

65 N. K. Hon, Z. Shaposhnik, E. D. Diebold, F. Tamanoi and B. Jalali, J. Biomed. Mater. Res., 2012, $100(12), 3416$.

66 M. A. Avidad, A. Nieto, A. Salinas-Castillo, L. Capitan-Vallvey, G. Miskelly and M. J. Sailor, Nano Res. Lett., 2014, 9(1), 1.

67 A. G. Cullis, L. T. Canham and P. D. J. Calcott, J. Appl. Phys., 1997, 82, 909.
68 B. Gelloz, Biodegradability of porous silicon, in Handbook of Porous silicon, Springer International Publishing, Switzerland, 2014, p. 307.

69 W. De Benedetti, S. Chiu, R. Radlinger, J. Ellison, B. A. Manhat, J. Z. Zhang and J. Shi, J. Phys. Chem. C, 2015, 119(17), 9595.

70 G. Ledoux, J. Gong, F. Huisken and O. Guillois, Appl. Phys. Lett., 2002, 80(25), 4834.

71 Y. Cai and Y. Lapitsky, Colloids Surf., B, 2014, 115, 100.

72 A. Loni, Porous silicon formation by anodization, in Handbook of Porous silicon, Springer International Publishing, Switzerland, 2014, p. 11. 